Title

A study of health professionals' views of the effectiveness of nutrition care in

$$
\text { general practice }
$$

\author{
Authors \\ Lauren Ball MND, Roger Hughes $P h D$, and Michael Leveritt $P h D$ \\ School of Public Health, Griffith University, Gold Coast, Australia. \\ Griffith Health Institute
}

\title{
Authorship disclosure
}

Lauren Ball conceptualised the study, conducted interviews, transcribed and analysed the data and took a lead role in drafting this manuscript. Roger Hughes contributed to the study design, data analysis and manuscript preparation. Michael Leveritt contributed to the study design, data analysis and manuscript editing. All authors participated in finalisation of the manuscript.

\section{ACKNOWLEDGEMENTS}

The time, insights and opinions shared by health professionals who participated in this study is appreciated. 


\section{Abstract}

Aim: This qualitative study aimed to explore the perceptions of key health professionals relating to the effectiveness of nutrition care provided in the general practice setting.

Methods: Twenty-eight health professionals across a range of disciplines (general practitioners $(n=11)$, practice nurses $(n=3)$, dietitians $(n=5)$, naturopaths $(n=5)$, and exercise physiologists $(n=4))$ individually participated in a semi-structured telephone interview, guided by an inquiry logic informed by the literature. Interviews were transcribed verbatim and analysed thematically using a constant comparison approach.

Results: Health professionals, including general practitioners, perceived that nutrition care provided in the general practice setting was mostly ineffective at improving patient nutrition behaviour. This was reported to be due to nutrition care competency deficits amongst general practitioners, a general practice reimbursement system that encourages practices inconsistent with quality nutrition care, and a low prioritisation of nutrition care in general practice. Tensions were apparent between health professional groups which may be hindering the successful implementation of interdisciplinary nutrition care for patients with chronic disease in this setting.

Conclusion: Without systematic changes to Australian primary health care model, the demand on general practitioners as primary providers of nutrition care will continue, therefore mandating support for general practitioners providing care in this context. Further research is required to identify strategies to improve nutrition care and opportunities to facilitate integrated health care provided to the general public within the general practice setting.

Key words: general practice, general practitioners, nutritional management, chronic disease 


\section{Introduction}

Chronic disease represents a substantial and increasing proportion of the burden of disease and associated health care expenditure in Australia. ${ }^{1,2}$ In 2007 more than $86 \%$ of Australians were reported to be suffering from one or more long-term health conditions, ${ }^{1}$ and over $81 \%$ of health care expenditure was allocated to chronic disease. ${ }^{2}$ Over two thirds of the risk factors for overweight/obesity, cardiovascular disease and Type II Diabetes are attributable to suboptimal nutrition. $^{3}$ The importance of optimal nutrition for chronic disease management is widely recognised by governing bodies such as the World Health Organisation, ${ }^{4,5}$ and nutrition care is included as a cornerstone for chronic disease management as outlined in current best practice guidelines. ${ }^{6}$ Nutrition care in this context can be viewed as any practice by a health professional to improve nutrition behaviour and subsequent health outcomes of an individual patient, including nutrition-related assessment, education and evaluation.

As a result of the increasing prevalence of chronic disease, the Australian general practice setting has experienced an increase in chronic disease presentation by patients; from 46.5 to 50.8 per 100 encounters in 2008-2009, resulting in an extra 4.8 million consultations per year. ${ }^{7,8}$ General practitioners (GPs) can be considered the 'gate keepers' of Australian primary care because they operate as the initial contact point for patients requiring non-emergency care, and are able to refer patients to health professionals for specialised treatment when required. An important referral pathway for patients with chronic disease is the Chronic Disease Management (CDM) Medicare scheme (previously known as Enhanced Primary Care). ${ }^{9}$ This relatively recent extension of Medicare reimbursement for patients consulting allied health professionals encourages GPs to plan and coordinate interdisciplinary, team-based health care for eligible patients with chronic conditions. An underlying assumption of this initiative is that an interdisciplinary approach to chronic disease 
management, including nutrition care, is the preferred mode of health service provision for optimal patient outcomes.

Accredited Practising Dietitians (APDs) are the only recognised professionals with assessed nutrition competencies, to provide nutrition care as part of the CDM Plan. In 2009 it was reported that 124 111 nutrition consultations were provided by APDs under the CDM initiative. ${ }^{9,}{ }^{10}$ Based on the number of full-time-equivalent APDs working in private practice settings, the maximum capacity for CDM nutrition consultations is approximately 630000 per annum. ${ }^{10}$ As a comparison, GPs currently discuss nutrition concepts in approximately $7 \%$ of consultations, which equates to over 7.9 million occurrences per year. ${ }^{7}$ This suggests that despite the previously stated assumption of preferred interdisciplinary nutrition care, GPs continue to constitute the gatekeepers of nutrition care in the Australian health system, therefore warranting further exploration of general practice based nutrition care.

Nutrition care can be provided outside the CDM Plan by a range of health professionals, irrespective of their formal nutrition competencies. However, role demarcation between these health professional groups is currently unclear. Furthermore, with current movements towards interdisciplinary care, the optimal interaction between health professionals providing nutrition care requires clarification.

Previous literature has explored GPs perceptions of their role in providing nutrition care, ${ }^{11,12}$ yet no known studies have examined the perspectives of a range of health professionals on the nutrition care provided by GPs. Exploring the views of health professionals involved in nutrition care may provide an understanding of the factors influencing nutrition specific interdisciplinary collaboration, perceptions of professional practice boundaries and opportunities for improved patient care. The aim of the current study was to explore the perceptions of health professionals regarding the 
effectiveness of nutrition care provided by GPs in the context of the Australian primary health care system. 


\section{Methods}

A semi-structured qualitative interview design was employed. Semi-structured interview questions were developed as open-ended questions to guide discussions. Interview questions were informed by a review of published literature using an inquiry logic that reflected the investigative aims of the study. Table 1 outlines each question, including the inquiry logic in relation to generation of information from participants.

\section{INSERT TABLE 1 ABOUT HERE}

The research team identified groups of health professionals whose professional bodies specifically claimed to have a role in the nutrition care of chronic disease management, and therefore likely to include nutrition in their service provision for patients with chronic disease. Identified health professional groups were GPs, practice nurses, APDs, naturopaths, and Accredited Exercise Physiologists (AEPs).

In order to access identified health professional groups, purposive non-probability sampling through invitation and self-selection was utilised (Table 2). Potential participants, including a professional group contact sourced from Association websites, were contacted via email and provided with a participant information sheet outlining the rationale and process of the interview. Participant recruitment continued until saturation of themes from each inquiry question was reached.

\section{INSERT TABLE 2 ABOUT HERE}

Each interview question was asked in an identical manner by author 1 , and no additional prompts were necessary. Interview duration averaged 17 minutes, with a range of 9 to 26 minutes. Interviews were audio-taped with participants' permission and transcribed by Author 1. 
Data analysis was conducted using a constant comparative approach to thematic analysis, including open and axial coding. ${ }^{13,} 14$ Firstly, author 1 manually coded sections of the transcripts and organised these into categories with common themes. Secondly, these themes were entered into a Microsoft Excel spreadsheet in order to link themes according to their properties and dimensions. ${ }^{13}$ Saturation of themes occurred when additional interviews did not produce new themes, with confirmation by authors 1 and 3 . Post analysis discussion and verification of themes were conducted between authors 1 and 3 to identify common or dissident viewpoints amongst interviewed participants. Original transcripts were edited grammatically to provide examples of key and/or contradicting themes.

The study protocol was reviewed and approved by the relevant university Ethics Committee. 


\section{Results}

A total of 28 individuals participated in the study. Their general characteristics are reported in Table

3. The average number of years in practice was 13.9 (range 4-42), however GPs reported considerably more years in practice (average 20.3 years) than allied health professionals (average 10.6 years). Nearly all participants $(25,93 \%)$ reported providing nutrition care to patients on a regular basis, and several allied health professionals $(10,59 \%)$ reported that they formally collaborated with GPs regarding nutrition care provision.

\section{INSERT TABLE 3 ABOUT HERE}

Table 4 summarises the key themes and sub-themes relating to health professionals perceptions of the effectiveness of nutrition care in general practice.

\section{INSERT TABLE 4 ABOUT HERE}

Despite many participants $(19 / 28,68 \%)$ stating that nutrition care is ideally within the scope of the GP's role in chronic disease management, most (including 8/11 GPs) viewed the effectiveness of such care in the GP setting as limited. This strong theme of ineffectiveness was moderated by another less common theme that the effectiveness of nutrition care varied greatly between GPs.

"Generally speaking I would say it's [nutrition care by GPs] a bit ordinary...I don't think on the whole GPs do it very well". (General Practitioner, 16 years experience)

Of the few interviewees $(2 / 28)$ who reported nutrition care as effective in the general practice setting, this rating was attributed to the role of the practice nurse, rather than the GP.

"General practice now is not just GPs; it's a team including practice nurses whom are often in practices doing the prevention, have a role in prevention and giving people advice about diet and other lifestyle issues". (General Practitioner, 15 years experience) 
Ineffectiveness tended to reflect a perception of superficiality of nutrition care, as articulated in the following response.

"It's [nutrition care] not that effective from my perspective. I think it's very generalised when patients visit a GP. They [GPs] don't have any nutrition background and [for] a lot of them, what they do is very generalised, a blanket approach.... and a lot of the time they [patients] come in and say 'oh my doctor said that I should do this' which is just something that they tell every client to do". (Exercise Physiologist, 6 years experience)

Interestingly, the participants' perceived ineffectiveness of nutrition care provided by GPs tended to be inferred by anecdotal reports of patient dissatisfaction with general practice nutrition care rather than objective evidence. Many of the allied health participants $(8 / 14,57 \%)$ described situations with patients who had previously seen a GP for nutrition care, and then proceeded to seek nutrition care elsewhere. Within these described experiences, two main themes emerged; patient confusion after receiving conflicting advice from health professionals (6/14,43\%), and patients' dissatisfaction with GP nutrition care $(5 / 14,36 \%)$.

"They've [patients] come to us and said 'Look I've just been to my GP, they've told me to lose weight, how do I do that?' or 'They've told me I need to eat better, what should I be eating?'...The GP hasn't given them the answer at all...so they [patients] often seek information elsewhere." (Exercise Physiologist, 6 years experience)

The limited training of GPs in nutrition care underscored the perception that nutrition care in general practice is ineffective.

"It [nutrition] doesn't seem to be much on the radar during university education...It's more of an afterthought or a little add on instead of a foundation of the training, so in the end they 
[GPs] leave university with very little knowledge about nutrition". (General Practitioner, 12 years experience)

Assertions of competency deficits did not necessarily imply that GPs were not interested in nutrition as a component of general practice care, with reports of some GPs demonstrating an active interest in nutrition and pursuing further education. However further education and interest in this field was not always consistent with evidence-based nutrition care for chronic disease management.

"They [GPs] don't have the knowledge. Some GPs who have gone off on a tangent, they're very devoted to nutrition but it's more alternative nutrition therapy...more about the Echinacea and herbal medicine, rather than what we know as nutrition therapy, and I think there are more and more GPs picking up that qualification and that interest". (Dietitian, 15 years experience)

The Medicare remuneration system was not viewed by interviewees as conducive to GPs spending adequate time with patients generally, and particularly in the context of providing effective nutrition care.

"If only we could have a remuneration model that would allow us to spend more time during opportunistic visits or actually schedule an appointment arising, let's say a full hour to really get the message across". (General Practitioner, 12 years experience)

Others criticised the lack of targeting of the Medicare CDM system towards early intervention for chronic disease, because it was dependent on patients seeking care from GPs once they were unwell, before being referred for nutrition care. This system seemed to perpetuate inefficient treatment approaches rather than prevention and early intervention. 
"Only patients who are already very sick can access them [MBS Chronic Disease Management Plans], instead of those where we can see that if we would do a little bit right now, they don't get very sick in the first place...Say for a prediabetic, there's just no way under Medicare that he can access a nutritionist, but 5 years down the track when everything has derailed and he's terribly sick, no problem we can do a care plan and he can get access. It's wrong". (General Practitioner, 12 years experience)

A sense of concern was clearly identified when participants discussed the cultural portrayal of GPs as all-knowing, despite perspectives of limited effectiveness of nutrition care provision.

"GPs are made to be the centre of everything, and they know it all. I'm not saying that's bad, but that's just the way it is. Whenever there's an advertisement, it always says 'check with your medical practitioner before starting this'. So it's actually promoted that the GP knows something about everything". (Dietitian, 15 years experience)

Nearly all interviewees $(25 / 28,89 \%)$ stated that a lack of time was the most significant determinant of the effectiveness of GPs in providing nutrition care. The words time, or lack of time were used 248 times by the 28 participants in relation to nutrition care provision by GPs, indicating a consistent theme throughout the interviews. Having inadequate time for patient interactions was reported to have numerous consequences influencing nutrition care effectiveness.

"I think GPs can be effective [at nutrition care], but when a patient sees a GP they will generally have their list of four or five problems and we get to sneak in a 20 or 30 seconds about diet - and often gets lost in all the other advice we're trying to give". (General Practitioner, 22 years experience) 
This issue of nutrition being a lower order priority was reported to be more evident in bulk billing practices.

"I think the biggest concern is that the majority of surgeries I work with are bulk billing surgeries, and their consultations are less than 10 minutes, and they try and turn patients around in that period of time. So I don't think they can facilitate appropriate care, and neither do they have the time to up skill their own knowledge in those areas". (Dietitian, 10 years experience)

Responses during interviews across disciplinary groups demonstrated a range of tensions that would appear to work against interdisciplinary care. A lack of awareness of the utility of, and roles of, different professionals was noticed amongst comments from GPs in the interviewee sample. Some GPs perceived the advice they provided as consistent with, and as effective as, dietitians.

"I think people [who] end up with dietitians realise that the advice wasn't that different to what the GP was telling them, just a little bit more specific". (General Practitioner, 22 years experience)

This view was at odds with a strong response theme suggesting that the most appropriate response from GPs to enhance nutrition care was to increase referral practices to nutrition specific allied health professionals. These health professionals were perceived to be less constrained by time and had more specific nutrition care competencies (such as dietitians and naturopaths). However, this common view amongst non-GP participants was disparate from GP participants, who tended to value be less enthusiastic about shared care.

"If we could delegate that [nutrition care] to another professional who could do it, that would be good. But you know, I think the impact is probably reduced, I think we probably 
need to investigate some alternative methods of imparting the information ourselves." (General Practitioner, 26 years experience)

There was a noticeable cynicism amongst some non-GP participants towards the nutrition care provided by GPs.

"I mean they're all talking about evidence based medicine, which is all very nice, but we are sitting through all of these complicated chemical medical scenarios because you've just been told by some rep who shouted you some seminar somewhere or a trip to the islands...really, it's hard to assume that they can even ask a person anything about their lifestyle or diet". (Naturopath, 6 years experience)

"At the end of the day they're my main business provider, so I don't want to try to belittle them with the information they don't know" (Dietitian, 8 years experience). 


\section{Discussion}

The aim of the current study was to explore the perceptions of key health professionals relating to the effectiveness of nutrition care provided in the general practice setting. Participants perceived that nutrition care provided in the general practice setting was largely ineffective due to a complex interaction of issues, ranging from individual barriers such as competency in practice, through to Medicare reimbursement system failures that distort health care provision. These perceptions are consistent with previous investigations of GP self-efficacy surrounding nutrition care ${ }^{11}$, as well as international studies examining the effectiveness of GP-facilitated lifestyle change programs which incorporate nutrition. ${ }^{15,16}$

Comments from the participant interviews highlight a common rationale provided for perceived patient dissatisfaction with GP-facilitated nutrition care, that nutrition care is superficial. This care is perceived as insufficient to effectively assist behaviour change. Superficiality of nutrition care has previously been linked to Australian GPs' view of their role in providing this care, and expectations that individualised care may be more appropriately provided by a nutrition-specific health professional, such as an Accredited Practising Dietitian. ${ }^{11}$ Primary reasons for this view include a perception that nutrition care may be more effective when provided by a health professional with nutrition specific competencies, and these health professionals are perceived as possessing the appropriate time required for patients. Despite these views of ineffectiveness, the current Medicare system positions GPs as the gatekeepers to the provision of nutrition care in primary health care. Without systematic changes to this model, the demand on GPs as primary providers of nutrition care will continue, therefore mandating support for GPs providing care in this context. 
Participant perceptions' regarding a lack of medical nutrition education received by GPs is consistent with international literature declaring that GPs undertake insufficient medical nutrition education to effectively promote healthy lifestyle and chronic disease management in their daily practice. ${ }^{17-19}$ The perception that GPs are under-prepared for nutrition care is reinforced by a recent study amongst Australian medical educators which suggests GPs are inadequately prepared for nutrition care practices required in the Australian general practice setting. ${ }^{11}$

The strong theme of a lack of time to provide nutrition care in GP consultations may infer that nutrition is low on the priority list for chronic disease management by GPs. The average length of a GP consultation in Australia is approximately 15 minutes. ${ }^{20}$ As a comparison, the recommended consultation lengths for nutrition care by APDs for patients with Type II Diabetes and overweight/obesity range from 20 to 60 minutes. ${ }^{21,} 22$ These evidence-based recommendations encompass time available to conduct a full diet history, anthropometry assessment and discussion of metabolic outcomes at each consultation. It may be inferred that the quality and/or quantity of nutrition care possible by GPs is limited by the restrictions of available time for consultation.

A lack of awareness of the utility of, and roles of, different professionals was noticed amongst comments from the interviewee sample. Furthermore, the research team noted underlying conflict and competitive attitudes during the conduction of participant interviews. This conflict appeared to mostly surround the ambiguous nature of respective health professional identities and subsequent health care roles. A number of response themes already reported from non-GPs in this study sample (such as ineffectiveness of nutrition care, nutrition competency deficits, forefront access to Medicare funds) clearly suggest negative attitudes about the Medicare system and the role of GPs in nutrition care. Competing interests suggests that the CDM Medicare items may not be promoting optimal chronic disease health care to patients, and may be hindering collaboration between health 
professionals. Further research is required to identify strategies to improve nutrition care provided to the general public within the current health care system.

The tensions identified through participant interviews highlight aspects of interdisciplinary care which requires further investigation. Current discussions about Australian health reform and subsequent chronic disease management assume interdisciplinary collaboration as a prerequisite for effective care, including nutrition specific care. It appears that current perceptions held by health professionals may be hindering the successful implementation of interdisciplinary nutrition care for patients with chronic disease.

The motive for allied health professionals expressing a desire for GPs to refer patients may be interpreted to enhance optimal patient outcomes. However this may also be interpreted as a vested interest in increased business generated through this referral system. These comments may also reflect an assumption held by allied health professionals who believe themselves to be more capable of providing nutrition care than GPs within the current health care system, despite limited evidence supporting this notion. ${ }^{23,24}$

There are two noteworthy limitations to this study. Firstly, variations in the geographical recruitment and recruitment methods of health professional groups may have influenced the perceptions provided by key health professionals.. Furthermore, recruitment based on self selection may have resulted in selection bias, meaning that the views of interviewed participants may not be reflective of the norms of these groups. Despite this, the views represented in this study demonstrate the need for further investigation of methods to enhance interdisciplinary nutrition collaboration. 
Further research is required to identify strategies to improve nutrition care and opportunities to facilitate integrated health care provided to the general public within the general practice setting.

\section{Acknowledgements}

The time, insights and opinions shared by health professionals who participated in this study is appreciated. 


\section{References}

1. Australian Institute of Health and Welfare. Incidence and prevalence of chronic diseases. Canberra 2007.

2. Australian Institute of Health and Welfare. Health system expenditure on chronic diseases. Canberra 2005.

3. Australian Institute of Health and Welfare. Prevalence of risk factors for chronic diseases. Canberra 2009.

4. National Health Priority Action Council (NHPAC). National Chronic Disease Strategy. Canberra: Australian Government Department of Health and Ageing 2006.

5. World Health Organization. Diet, nutrition, and the prevention of chronic diseases. Geneva WHO Technical Report Series 1990.

6. Diabetes Australia, Royal Australian College of General Practitioners. Diabetes Management in General Practice. Fourteenth Edition. Sydney 2008.

7. Britt H, Miller G, Knox S. General practice activity in Australia 2008-09. General Practice Series. Canberra Australian Institute of Health and Welfare 2010.

8. Australian Institute of Health and Welfare. General practice in Australia, health priorities and policies 1998-2008. Canberra: Australian Institute of Health and Welfare 2009.

9. Medicare Australia. Medicare Benefits Schedule. Canberra: Department of Health and Ageing 2010.

10. Mitchell L, Capra S, MacDonald-Wicks L. Structural change in Medicare funding: impact on the dietetics workforce. Nutr Diet. 2009;66(3):170-5.

11. Ball L, Hughes R, Leveritt M. Nutrition in General Practice: Role and workforce preparation expectations of medical educators. AJPH. 2010;16(4):304-10. 
12. Pomeroy SEM, Worsley A. Nutrition care for adult cardiac patients: Australian general practitioners' perceptions of their roles. J Fam Prac. 2008;25:I123-19.

13. Strauss A, Corbin J. Basics of Qualitative Research. 2nd Edition. California: SAGE Publications; 1998.

14. Thorne S. Data analysis in qualitative research. 2000 July 1, 2000;3(3):68-70.

15. Ashenden R, Silagy C, Weller D. A systematic review of the effectiveness of promoting lifestyle change in general practice. J Fam Prac. 1997;14(2):160-76.

16. Martin PD, Dutton GR, Rhode PC, Horswell RL, Ryan DH, Brantley PJ. Weight loss maintenance following a primary care intervention for low-income minority women. Obesity. 2008;16(11):2462-7.

17. Adams KM, Kohlmeier M, Zeisel SH. Nutrition Education in U.S. Medical Schools: Latest Update of a National Survey. Acad Med. 2010;85(9):1537-42.

18. Powell-Tuck J. Nutrition in medical education. JRSM Short Rep. 1983;76(12):997.

19. Orimo H, Shimura T, Shimada T. Nutrition education in medical schools in Japan: results from a questionnaire survey. Asia Pac J Clin Nutr. 2006;15(3):323-8.

20. Australian Institute of Health and Welfare. General practice workforce and workload. General practice in Australia, health priorities and policies 1998-2008. Sydney: Family Medicine Research Centre 2008.

21. Dietitians Association of Australia. Evidence Based Practice Guidelines for the Nutritional Management of Type 2 Diabetes Mellitus for Adults. Sydney 2006.

22. Dietitians Association of Australia. Best Practice Guidelines for the Treatment of Overweight \& Obesity in Adults. Canberra 2004.

23. Ashley JM, St Jeor ST, Schrage JP, Perumean-Chaney SE, Gilbertson MC, McCall NL, et al. Weight control in the physician's office. Arch Intern Med. 2001;161(13):1599-604. 
24. Willaing I, Ladelund S, Jorgensen T, Simonsen T, Nielsen LM. Nutritional counselling in primary health care: a randomized comparison of an intervention by general practitioner or dietician. Eur J Cardiovasc Prev Rehabil. 2004;11(6):513-20.

25. Yellow Pages. Find A Naturopath in Your Location. 2010; Available from: www.yellowpages.com.au/naturopath accessed 10 September 2010.

26. Medibank Private. Find a Health Care Provider. 2010; Available from: www.medibank.com.au/Client/StaticPages/FindProvider.aspx accessed 10 September 2010. 
Please tell me about your experience and current involvement in the domain of general practice.

Identify experiences important to the development of perceptions and viewpoints regarding nutrition care and general practice.

How effective do you think nutrition care in the general practice setting is currently?

Identify perceived feasibility of effective nutrition care practices

How effective do you think nutrition care in the general practice setting in general practice. potentially is?

To what extent do you think that general public patients perceive general practitioners as having expertise in the field of nutrition care?

Determine what the health professional perceives the influence of patient expectations is on nutrition care practices.

To what extent do you think general practitioners feel competent in providing nutrition care?

Explore the feasibility of nutrition care provision by GPs in the general practice setting.

To what extent do you think that GPs are competent for nutrition care in the general practice setting?

Consider perceptions of health professionals on GP competency levels based on their experiences.

What are some of the barriers to nutrition care provision in the general practice setting?

Consider perceptions of health professionals regarding What are some of the opportunities to provide further nutrition care provision in the general practice setting? Can you think of any strategies to act on these opportunities?

Do you have anything else you would like to add?

Provide opportunity for open expression of views of the health professional. 
Table 2 - Contact method for respective professional groups

\begin{tabular}{ll}
\hline Professional Group & Contact Method \\
\hline Accredited Exercise & Convenience and snowball sampling of exercise physiologists \\
Physiologists & $\begin{array}{l}\text { working in a private practice or general practice capacity were } \\
\text { already available to researcher, or provided by word of mouth. }\end{array}$ \\
Accredited Practising & $\begin{array}{l}\text { Introductory email sent out through the Dietitians Association of } \\
\text { Dietitians }\end{array}$ \\
Australia national Private Practice Interest Group list serve. \\
Interested participants were asked to contact LB individually. \\
A list of medical centres and contact details were accessed \\
through the Queensland Yellow Pages Online. ${ }^{25}$ Calls to medical \\
centres were conducted to obtain email address of practice \\
manager, who was then asked to forward the email to general \\
practitioners and practice nurses in each clinic. \\
Email address accessed through publically available information \\
accessed online via Medibank Private Queensland Naturopath \\
Listing, ${ }^{26}$ and Queensland Yellow Pages Online Listing.
\end{tabular}


Table 3: Participant Characteristics

\begin{tabular}{|c|c|c|c|c|}
\hline Participant Group & $\begin{array}{l}\text { Number } \\
\text { (n) }\end{array}$ & $\begin{array}{l}\text { Years in Practice } \\
\text { (Average; Range) }\end{array}$ & $\begin{array}{l}\text { Provide Nutrition Care to } \\
\text { Patients on a Regular Basis }\end{array}$ & $\begin{array}{c}\text { Formal Collaboration with } \\
\text { GPs re: Nutrition Care of } \\
\text { Patients }\end{array}$ \\
\hline Accredited Exercise Physiologists & 4 & $6.0(3-10)$ & $4 / 4(100 \%)$ & $1 / 4(25 \%)$ \\
\hline Accredited Practising Dietitians ${ }^{a}$ & 5 & $13.4(4-30)$ & $5 / 5(100 \%)$ & $5 / 5(100 \%)$ \\
\hline General Practitioners ${ }^{b, c, d}$ & 11 & $20.3(15-42)$ & $9 / 10(90 \%)$ & N/A \\
\hline Naturopaths & 5 & $11.5(6-21)$ & $5 / 5(100 \%)$ & $2 / 5(40 \%)$ \\
\hline Practice Nurses ${ }^{\mathrm{e}}$ & 3 & $17.0(8-23)$ & $2 / 3(67 \%)$ & $2 / 3(67 \%)$ \\
\hline Total & 28 & $13.9(4-42)$ & $25 / 27(93 \%)$ & $10 / 17(59 \%)$ \\
\hline
\end{tabular}

$a=$ Including a representative of the Dietitians Association of Australia.

$b=$ Including a representative of the Australian Medical Association

$c=$ Including a representative of the Gold Coast Division of General Practice

$d=$ Including a representative of the General Practice Education Training Program (administered by the Royal Australian College of General Practitioners),

however is not a GP themselves, and practice demographic has been excluded from calculations.

$e=T$ wo of the practice nurses also worked as practice managers. 
Table 4: Key response themes relating to the effectiveness of nutrition care in general practice

\begin{tabular}{|c|c|}
\hline Theme & Sub-theme \\
\hline \multicolumn{2}{|l|}{$\begin{array}{l}\text { Nutrition care provided by } \\
\text { GPs is ineffective }\end{array}$} \\
\hline & Nutrition care in general practice is superficial and variable \\
\hline & Observations of patient dissatisfaction suggest ineffective practice \\
\hline \multicolumn{2}{|c|}{$\begin{array}{l}\text { GPs have nutrition care } \\
\text { competency deficits }\end{array}$} \\
\hline & Inadequate GP education in nutrition care \\
\hline & A lack of intraprofessional awareness \\
\hline & Some GPs are interested and passionate about nutrition \\
\hline \multicolumn{2}{|c|}{$\begin{array}{l}\text { Medicare reimbursement } \\
\text { distorts service delivery }\end{array}$} \\
\hline & $\begin{array}{l}\text { Medicare reimbursement incentivizes short consultations creating } \\
\text { time poverty in general practice }\end{array}$ \\
\hline & $\begin{array}{l}\text { Frustrations regarding privileged access and control of Medicare } \\
\text { reimbursement by GPs working against interdisciplinary care }\end{array}$ \\
\hline \multicolumn{2}{|r|}{ 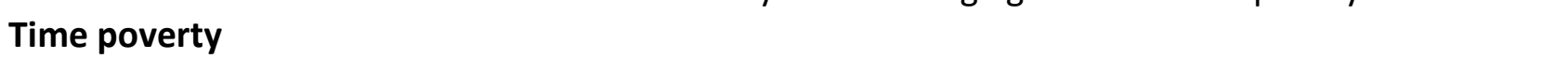 } \\
\hline & $\begin{array}{l}\text { Observations of patient dissatisfaction related to superficial care } \\
\text { Inadequate time to effectively provide nutrition care }\end{array}$ \\
\hline & $\begin{array}{l}\text { Time poverty forces prioritisation of issues, nutrition often lower } \\
\text { order priority, often neglected. }\end{array}$ \\
\hline \multicolumn{2}{|l|}{ Interdisciplinary tensions } \\
\hline & $\begin{array}{l}\text { Professional chauvinism evident about the most effective nutrition } \\
\text { care provider, despite limited evidence. }\end{array}$ \\
\hline & $\begin{array}{l}\text { Frustrations regarding privileged access and control of Medicare } \\
\text { reimbursement by GPs working against interdisciplinary care }\end{array}$ \\
\hline & Vested interests may moderate attitudes \\
\hline & A lack of interprofessional awareness limiting active referral \\
\hline
\end{tabular}

